\title{
A profissão farmacêutica sob a perspectiva de estudantes do ensino médio em Samambaia, Brasília, DF, Brasil
}

\author{
The pharmaceutical profession under the perspective of \\ students of high school in Samambaia, Brasilia, DF, Brazil
}

Recebido em: 09/04/2019 Aceito em: $\quad 30 / 07 / 2019$

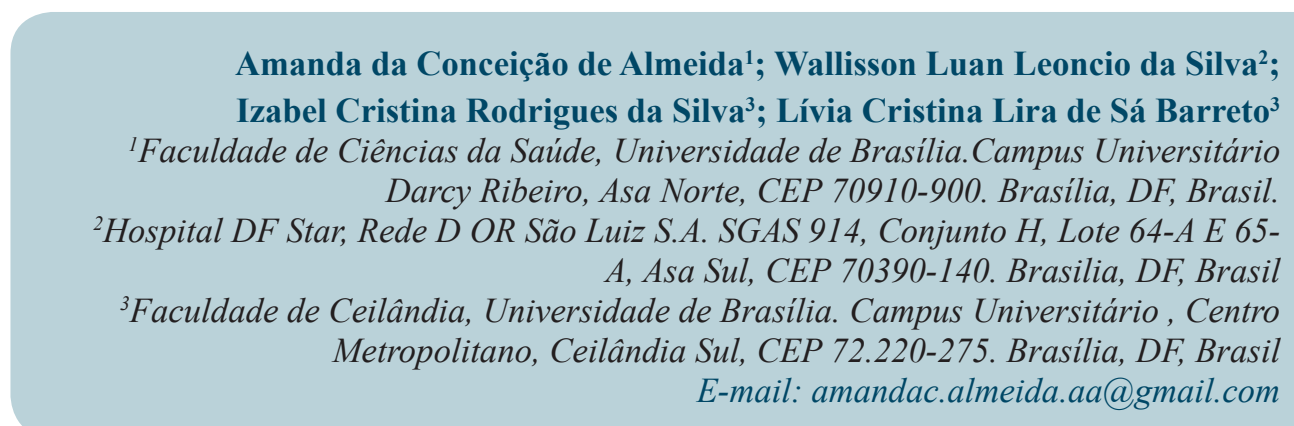

ABSTRACT:

The undergraduate courses of Pharmacy in Brazil allow insertion in the market of a professional with generalist characteristics who dominates the composition and production of medicines, food, and cosmetics, can provide pharmaceutical assistance, and receives training in clinical and toxicological analyzes, including humanistic, ethical, technical and scientific profile. Despite the complex and important rule of this professional in the health area, beginner's pharmacy students are mostly unaware of most of the areas that the pharmacist can be. Thus, the present study aimed to apply an active methodology for the dissemination of knowledge about the pharmaceutical profession. Therefore, a study was conducted with 24 high school students in Samambaia - DF. A questionnaire was applied to the volunteers before the exposure of audiovisual material containing interviews, texts, and images about the pharmaceutical profession, and another questionnaire was applied after this exhibition. The first questionnaire evaluated the volunteer's profile, expectations of acting in the professional market and interest in the health area. The second questionnaire estimated the appropriation of knowledge about the pharmaceutical profession and interest in Pharmacy. The study allowed to measure the interest in the graduation in Pharmacy of higher school students and evidenced the lack of knowledge of the importance of the pharmacist for the health of the community.

KeyWords: students; higher education; pharmacists; societies; population health.

\section{RESUMO}

Os cursos de graduação em Farmácia no Brasil permitem a inserção no mercado de um profissional com caráter generalista que domina a composição e produção dos medicamentos, alimentos e cosméticos, presta assistência farmacêutica, possui formação em análises clínicas e toxicológicas, com perfil humanista, ético, técnico e científico. Apesar da capacitação complexa e importante para a saúde, os ingressantes nos cursos 
de Farmácia desconhecem, na maioria das vezes, a totalidade das áreas de atuação e impactos benéficos. Dessa maneira, o presente estudo visou a aplicação de metodologia ativa para a disseminação de conhecimento acerca da profissão farmacêutica. Para tanto, o estudo foi realizado com 24 acadêmicos do ensino médio em Samambaia-DF. Um questionário foi aplicado aos voluntários antes da exposição de um material audiovisual contendo entrevistas, textos e imagens sobre a profissão farmacêutica, e um outro questionário disponibilizado após visualização. O primeiro questionário avaliou o perfil do voluntário, suas expectativas de atuação no mercado de trabalho e interesse na área da saúde. O segundo questionário estimou a apropriação de conhecimento acerca da profissão farmacêutica e interesse pela Farmácia. $O$ estudo permitiu mensurar o desejo pela graduação em Farmácia de possíveis ingressantes no ensino superior e evidenciou o desconhecimento da importância do Farmacêutico para a saúde da comunidade.

PALAVRAS-CHAVE: estudantes; ensino superior; farmacêuticos; sociedades; saúde da população.

\section{INTRODUÇÃO}

A busca por tratamentos dos seus males é antiga e baseada no ato instintivo e empírico dos recursos naturais, observando e imitando os animais (1). As profissões médicas e farmacêuticas eram reconhecidas como detentoras de conhecimento para alcançar cura e paz, desenvolvendo atividades e se entrelaçando na busca pela saúde e cura dos sofrimentos físicos decorrentes de enfermidades (2). No entanto, o avanço do comércio de especiarias, a formação técnica sobre a fabricação de medicamentos e o desenvolvimento do ensino universitário da medicina acarretaram, como consequência, o afastamento progressivo dos médicos das atividades manuais, como a preparação de medicamentos, o que possibilitou o crescimento do número de boticários e a dissociação entre as duas profissões que seguiram para a separação legal (2).

A separação das profissões médica e farmacêutica proporcionou maior reconhecimento $\mathrm{e}$ prestígio ao farmacêutico possibilitando a criação e expansão das boticas - primeiras farmácias (3). As boticas possuíam duas áreas, uma para preparação e outra para comercialização das fórmulas farmacêuticas. Os insumos destinados às formulas medicamentosas eram armazenados em potes de cerâmica e ficavam à mostra (1). Nestes locais também aconteciam reuniões científicas, literárias e políticas com outros profissionais notáveis da cidade (3).

A separação das profissões médica e farmacêutica foi acompanhada da criação das Escolas de Farmácia, que desenvolviam primariamente suas atividades em estruturas anexas às faculdades de Medicina (2,3).
A primeira escola superior do curso de graduação em Farmácia que funcionou independente do curso de medicina surgiu na França no século XVI $(2,3)$.

No Brasil, a primeira Escola de Farmácia independente, foi criada em 1839, na cidade de Ouro Preto. Em 1972 já existiam 25 faculdades de Farmácia em todo Brasil (3).

Com o surgimento de novas doenças, as revoluções e as guerras impulsionaram a industrialização de medicamentos e sua produção em maior escala, ganhando lugar das fórmulas manipuladas (4). No Brasil, em adição à influência da Revolução Industrial, o desprezo da sociedade pelo farmacêutico foi estimulado pela cessão de funcionamento das boticas a indivíduos sem a devida formação, como por exemplo, comerciantes de secos e molhados, muitas vezes analfabetos, que visavam apenas o aumento de vendas e lucros $(3,4)$.

As transformações que acometeram os farmacêuticos e acadêmicos de Farmácia resultaram na mudança do currículo acadêmico e na permanência apenas da capacitação industrial e laboratorial, afastando ainda mais o profissional do contato direto com a sociedade (4). Contudo, por influência de movimentos estudantis, de farmacêuticos atuantes em diversas áreas como, homeopatia, farmácia hospitalar e vigilância sanitária, e com as atuais Diretrizes Curriculares Nacionais do Curso de Graduação em Farmácia, a formação do profissional visa habilitá-lo para ter conhecimento do todo, visão crítica e reflexiva, reconhecendo a condição 
humana, científica e intelectual, sendo apto a exercer atividades com ética e discernimento das características sociais, culturais e econômicas da população, com o propósito de beneficiá-la $(4,5)$.

Consoante com o Decreto $N^{\circ} 85878$ de 7 de abril de 1981 do Conselho Federal de Farmácia - CFF, os farmacêuticos graduados no Brasil possuem mais de 70 diferentes áreas de atuação entre atribuições privativas e outras atividades afins ao profissional (6), que são agrupadas, segundo a Resolução No 572 de 25 de abril de 2013 do Conselho Federal de Farmácia - CFF, em 10 especialidades: alimentos, análises clínico-laboratoriais, educação, farmácia, farmácia hospitalar e clínica, farmácia industrial, gestão, práticas integrativas e complementares, saúde pública e toxicológica (7).

No Brasil, até o ano de 2015, havia 447 instituições de ensino superior em Farmácia, segundo o Ministério da Educação (8).

$\mathrm{Na}$ diversidade dos cursos de graduação em farmácia disponibilizados pelo Brasil, há o ingresso, em cada semestre letivo, de acadêmicos que, na maioria das vezes, seleciona este curso sem o conhecimento profundo da atuação do profissional farmacêutico, desconhecendo, portanto os impactos dessa profissão para a população.

Essa carência de conhecimento leva à desistência do curso logo nos primeiros semestres letivos. Em vista disso, o presente trabalho avaliou o grau de conhecimento desta profissão por estudantes do terceiro ano do Ensino Médio.

\section{MÉTODO}

O projeto foi previamente submetido ao comitê de ética da Faculdade de Ciências da Saúde da Universidade de Brasília - CEP/FS-UnB (CAAE - Certificado de Apresentação para Apreciação Ética: 44949415.7.0000.0030 e Número do Parecer: 1.182.887), contendo o Termo de concordância de instituição co-participante, assinado pelo diretor responsável pela escola privada.

Os critérios de inclusão presentes na proposta para seleção dos voluntários participantes foram: alunos regularmente matriculados na instituição, com idade mínima de 13 anos, com consentimentos dos pais; alunos maiores de 18 anos que assinaram próprio termo de consentimento livre e esclarecido. O critério de exclusão foi aplicado apenas aos estudantes inaptos a responder os questionários escritos.

Também foi destacado o esclarecimento realizado pelo pesquisador orientador quanto à liberdade, por parte do voluntário, de desistir da entrevista em qualquer momento da pesquisa.

Foram aplicados dois questionários a 24 voluntários da escola do Ensino Médio em Samambaia (Brasília, DF), em março de 2016. Um questionário foi disponibilizado antes da exposição de um video contend informações e imagens sobre a profissão farmacêutica e curso de graduação em Farmácia na Universidade de Brasília, e o outro logo após.

Cada questionários apresentava cinco questões; o primeiro abordava o perfil dos voluntários e os cursos de graduação de interesse na área da saúde, além de relacionar as razões para a escolha e seu conhecimento na área da saúde, em especial, sobre Farmácia. O segundo questionário, continha questões que averiguavam a qualidade das informações apresentadas no video e verificava se o material exposto possibilitou despertar o interesse pelo curso de graduação em Farmácia. O material áudio-visual apresentado faz parte do projeto de extensão intitulado "Farmacêutico: profissional de grande impacto para a sociedade", com vigência na UnB desde 2013.

Este material, planejado, produzido e editado pela equipe executora do projeto de extensão mencionado previamente, contém informações sobre as áreas de atuação do farmacêutico, entrevistas com estudantes dos cursos de Farmácia da UnB e farmacêuticos atuantes em vários setores. $\mathrm{O}$ vídeo também apresenta informações quanto ao mercado de trabalho e as habilidades desejáveis para esta capacitação, refletindo a importância do farmacêutico e de seu papel na sociedade.

Os dados coletados foram adicionados em um banco de dados SPSS versão 22.0. As frequências das repostas foram descritas em termos absolutos e relativos. 


\section{RESULTADOS E DISCUSSÃO}

No primeiro questionário foi avaliado o perfil dos estudantes e suas expectativas com relação a sua atuação no mercado de trabalho e realização de cursos de graduação, buscando avaliar o interesse pela área de saúde e, em especial, pelo curso de Farmácia (Tabela 1).

A aplicação do questionário anterior à apresentação do vídeo destacou que a maioria dos voluntários era do sexo feminino $(79,2 \%)$, e o interesse pela área de Saúde $(66,7 \%)$. Porém foi evidenciada a falta de interesse pelo curso de Farmácia, ao solicitar aos entrevistados que elencassem os cursos prioritários para escolha. Apenas graduação em Medicina (31,3\%), Psicologia (18,9\%), Medicina Veterinária (12,5\%), Nutrição $(12,5 \%)$, Enfermagem $(6,2 \%)$, Odontologia (6,2\%), Biomedicina $(6,2 \%)$ e o curso de Técnico em enfermagem
$(6,2 \%)$ foram listados. Os fatores de escolha foram motivados principalmente por remuneração, em conjunto com outras motivações, como vocação e prestígio $(62,5 \%)$ das profissões listadas.

A diferença participativa entre os sexos é uma crescente histórica com o aumento significativo da participação feminina no ensino superior e nas diversas áreas de atuação, buscando carreiras de maior notoriedade (10).

Os fatores motivacionais podem ser caracterizados pelo interesse, satisfação e compensação pela concretização de uma escolha, suprindo dessa forma as motivações inerentes a si - intrínsecas, como também do convívio social - extrínsecas. Assim, os participantes acreditam que suas escolhas prioritárias de curso lhes proporcionarão profissões que resultem em vantajosa remuneração combinada com reconhecimento profissional e crescimento pessoal (11).

Tabela 1. Dados obtidos com o questionário 1 aplicado antes da exposição do video aos alunos do Ensino Médio em Samambaia (Brasília, DF) em março de 2016

\begin{tabular}{|c|c|c|c|}
\hline Questões & Respostas & N & $(\%)$ \\
\hline Gênero & $\begin{array}{l}\text { Feminino } \\
\text { Masculino }\end{array}$ & $\begin{array}{l}19 \\
5\end{array}$ & $\begin{array}{l}(79,2) \\
(20,8)\end{array}$ \\
\hline Interesse pela área da Saúde & $\begin{array}{l}\text { Sim } \\
\text { Não }\end{array}$ & $\begin{array}{l}16 \\
8\end{array}$ & $\begin{array}{l}(66,7) \\
(33,3)\end{array}$ \\
\hline Curso que pretende cursar após a conclusão do Ensino Médio & $\begin{array}{l}\text { Medicina } \\
\text { Psicologia } \\
\text { Medicina Veterinária } \\
\text { Nutrição } \\
\text { Biomedicina } \\
\text { Enfermagem } \\
\text { Odontologia } \\
\text { Técnico em Enfermagem }\end{array}$ & $\begin{array}{l}5 \\
3 \\
2 \\
2 \\
1 \\
1 \\
1 \\
1\end{array}$ & $\begin{array}{r}(31,3) \\
(18,9) \\
(12,5) \\
(12,5) \\
(6,2) \\
(6,2) \\
(6,2) \\
(6,2)\end{array}$ \\
\hline Motivação para escolha do curso pós Ensino Médio & $\begin{array}{l}\text { Vocação } \\
\text { Prestígio } \\
\text { Motivos diversos }\end{array}$ & $\begin{array}{l}5 \\
1 \\
10\end{array}$ & $\begin{array}{r}(31,3) \\
(6,2) \\
(62,5)\end{array}$ \\
\hline Que profissional considera de maior importância para a saúde & $\begin{array}{l}\text { Professor } \\
\text { Médico } \\
\text { Policial }\end{array}$ & $\begin{array}{l}14 \\
9 \\
1\end{array}$ & $\begin{array}{r}(58,3) \\
(37,5) \\
(4,2)\end{array}$ \\
\hline Que profissional presta informações sobre medicamentos? & $\begin{array}{l}\text { Farmacêutico } \\
\text { Médico }\end{array}$ & $\begin{array}{l}14 \\
10\end{array}$ & $\begin{array}{l}(58,3) \\
(41,7)\end{array}$ \\
\hline Qual profissional produz medicamentos? & $\begin{array}{l}\text { Biomédico } \\
\text { Farmacêutico }\end{array}$ & $\begin{array}{l}17 \\
7\end{array}$ & $\begin{array}{l}(70,8) \\
(29,2)\end{array}$ \\
\hline Qual profissional que realiza exames laboratoriais? & $\begin{array}{l}\text { Biomédico } \\
\text { Médico } \\
\text { Enfermeiro } \\
\text { Farmacêutico }\end{array}$ & $\begin{array}{l}13 \\
6 \\
4 \\
1\end{array}$ & $\begin{array}{r}(54,2) \\
(25,0) \\
(16,7) \\
(4,2)\end{array}$ \\
\hline
\end{tabular}


Quando questionados sobre o profissional que exerce maior influência na saúde da população, o Professor (58,3\%) e o Médico (37,5\%) foram designados, pela maioria dos participantes. Porém, a evidência de desconhecimento sobre as atribuições dos diferentes profissionais da área da Saúde, em especial, do farmacêutico, foi destacada nas perguntas relativas às atividades do profissional farmacêutico, como produzir medicamentos e realizar exames. Dos participantes, $70,8 \%$ responderam inadequadamente ser competência do profissional Biomédico a produção de medicamentos, enquanto que a realização de exames, também de forma equivocada, foi respondida como função de Médicos $(25,0 \%)$ e Enfermeiros $(16,7 \%)$.

De acordo com o Decreto 85878, de 7 de abril de 1981 (6), a produção de medicamentos é competência privativa do profissional farmacêutico, o qual, nas indústrias farmacêuticas, é o responsável técnico. Também contribuem para diagnósticos, que são empregados para tratamento e que pos- sam causar dependência mental, comportamental e física.

A realização de exames laboratoriais também é de competência do farmacêutico, como disposto no decreto acima citado, porém, não é uma atividade de âmbito privativo. Dessa forma, pode ser exercida por outros profissionais que são aptos e regulamentados por decretos e resoluções de acordo com cada conselho de classe, como é o caso dos profissionais Biomédicos e Biólogos, que atendem, respectivamente, à Resolução $\mathrm{N}^{\circ} 78$, de 29 de abril de 2002 (12), e à Resolução $\mathrm{N}^{\circ} 227$, de 18 de agosto de 2010 (13). A realização de exames laboratoriais não é caracterizada como atribuições dos enfermeiros e médicos como afirmado no Decreto $\mathrm{N}^{\circ} 94.406$, de 08 de junho de 1987 , e na Lei $\mathrm{N}^{\circ}$ 12.842, de 10 de julho de $2013(14,15)$.

O segundo questionário permitiu avaliar se o vídeo utilizado era adequado para a divulgação da profissão farmacêutica, captando sugestões para melhoria do material de exposição. Os dados estão apresentados na Tabela 2.

Tabela 2. Respostas do questionário 2 aplicado após a exposição do material audiovisual aos alunos do Ensino Médio em Samambaia (Brasília, DF), em março de 2016

\begin{tabular}{|c|c|c|c|c|}
\hline \multirow{2}{*}{ Perguntas } & \multicolumn{4}{|c|}{ Respostas } \\
\hline & Sim & $(\%)$ & Não & $(\%)$ \\
\hline Despertou interesse pela profissão farmacêutica & 5 & $(20,8)$ & 19 & $(79,2)$ \\
\hline Possibilitou conhecimento sobre a profissão farmacêutica & 24 & $(100,0)$ & . & . \\
\hline A sociedade precisa do profissional farmacêutico & 24 & $(100,0)$ & . & . \\
\hline Tempo de vídeo & 24 & $(100,0)$ & . & . \\
\hline Utilidade do vídeo & 24 & $(100.0)$ & . & . \\
\hline
\end{tabular}

O segundo questionário evidenciou a necessidade de inserir temáticas sobre assuntos a serem compreendidos, pois, apesar de ter despertado pouco interesse pela profissão farmacêutica $(20,8 \%)$, os estudantes afirmaram que o vídeo possibilitou conhecimento e destacou a importância do profissional farmacêutico. Assim, a finalidade do uso de material audiovisual como mediador do processo ensino-aprendizado foi alcançada, sendo um recurso didático capaz de inserir temáticas, ilustrações, expor conceitos e fazer leituras sobre assuntos a serem compreendidos (16).
O estudo traz algumas limitações, dentre elas, a exposição do material audiovisual e a aplicação dos questionários terem sido realizados em apenas uma instituição de ensino privado, e com isso, um número pequeno de amostra. Ainda assim, os dados apresentados pela pesquisa representam um alerta, requerendo atenção e maior intervenção na promoção da valorização do Curso e do farmacêutico. São necessários mais ações e projetos nas escolas e na sociedade a fim de difundir o mercado de atuação, despertar o interesse de futuros acadêmicos e conscientizar a população. 


\section{CONCLUSÃO}

A presente pesquisa evidenciou o desconhecimento da atuação farmacêutica, ainda que por uma parcela pequena da população. A situação é preocupante, ao considerar a falta de conhecimento, por parte dos participantes, também sobre os demais cursos de graduação relacionados ao curso de Farmácia, como Medicina, Biomedicina e Enfermagem. Esta falta de informação pode consistir um fator desmotivador de escolha e permanência nos cursos de graduação em Saúde.

O material audiovisual utilizado promoveu o conhecimento acerca da profissão farmacêutica e conscientizou da necessidade do farmacêutico para a sociedade. Sendo assim, o presente trabalho evidenciou a necessidade de atuação do profissional farmacêutico e execução de projetos de extensão nas escolas de Ensino Médio para difundir seu mercado de atuação, despertar o interesse de futuros acadêmicos e conscientizar a população.

\section{AGRADECIMENTOS}

Os autores agradecem à Universidade de Brasília pelo incentivo institucional e ao Conselho Nacional de Desenvolvimento Científico e Tecnológico - $\mathrm{CNPq}$ pelo apoio financeiro à pesquisa por meio do programa de iniciação científica ProIC/ $\mathrm{DPP} / \mathrm{UnB}$; ao comitê de ética pelo parecer favorável, aos discentes e farmacêuticos que participaram da produção do material audiovisual e a instituição privada e seus alunos que permitiram o desenvolvimento do trabalho

\section{REFERÊNCIAS}

1. Aiache JM, Aiache S, Renoux R. Iniciação ao conhecimento do medicamento. 2. ed. São Paulo: Organização Andrei; 1998. 380p.

2. Dias JPS. A Farmácia e a História: Uma introdução à História da Farmácia, da Farmacologia e da Terapêutica. Lisboa: Faculdade de Farmácia da Universidade de Lisboa; 2005. [Acesso em: 10 Abr 2019]. Disponível em: http://leg.ufpi.br/subsiteFiles/lapnex/arquivos/files/Farmacia-e-Historia.pdf

3. Santos JD, Silva JAP. Conselhos de Farmácia: Memória e Prospecção. Brasília: Cidade Gráfica e Editora Ltda, 2003. 145p

4. Lorandi PA. Análise Histórica da Formação Acadêmica do Farmacêutico - Quatro Décadas. Infarma. 2006; 8(7/8): 7-12.

5. BRASIL. Resolução CNE/CES 2, de 19 de fevereiro de 2002. Institui Diretrizes Curriculares Nacionais do Curso de Graduação em Farmácia. Ministério da Educação. Diário Oficial da União 04 Mar 2002; Seção 1. 9p.

6. BRASIL. Decreto 85878 de 7 de abril de 1981. Estabelece normas para execução da Lei $\mathrm{n}^{\circ} 3.820$, de 11 de novembro de 1960, sobre o exercício da profissão de farmacêutico, e dá outras providências. Diário Oficial da União 07 Abr 1981; Seção 1. 6631 p.

7. CFF. Resolução No 572 de 25 de abril de 2013. Dispõe sobre a regulamentação das especialidades farmacêuticas, por linhas de atuação Conselho Federal de Farmácia.

8. BRASIL. Ministério da Educação. Instituições de Educação Superior e Cursos Cadastrados. [Acesso em: 10 Abr. 2019]. Disponível em: http://emec.mec.gov.br/.

9. CFF. Resolução $\mathrm{N}^{\circ} 471$, de 28 de fevereiro de 2008. Aprova o regulamento sobre os símbolos oficiais dos farmacêuticos. Conselho Federal de Farmácia.
10. Guedes MC. A presença feminina nos cursos universitários e nas pós-graduações: desconstruindo a idéia da universidade como espaço masculino. História, Ciências, Saúde - Manguinhos. Jun 2008; 15:117-132. DOI: 10.1590/S0104-59702008000500006.

11. Araújo MV, Silva JWB, Franco EM. Motivação para o aprendizado em estudantes de graduação em Psicologia. Rev Psicol: Teoria e Prática. 2014;16(2):185-198. DOI: 10,15348/1980-6906/psicologia.v16n2p185-198.

12. CFBM. Resolução $N^{\circ} 78$, de 29 de abril de 2002. Dispõe sobre o Ato Profissional Biomédico, fixa o campo de atividade do Biomédico e cria normas de Responsabilidade Técnica. Conselho Federal de Biomedicina

13. CFBIO. Resolução N²27, de 18 de agosto de 2010. Dispõe sobre a regulamentação das Atividades Profissionais e as Áreas de Atuação do Biólogo, em Meio Ambiente e Biodiversidade, Saúde e, Biotecnologia e Produção, para efeito de fiscalização do exercício profissional. Conselho Federal de Biologia.

14. BRASIL. Decreto $N^{\circ} 94.406$, de 08 de junho de 1987. Regulamenta a Lei $\mathrm{N}^{\circ} 7.498$, de 25 de junho de 1986, que dispõe sobre o exercício da Enfermagem, e dá outras providências. Diário Oficial da União 09 Jun 1987; Seção $1.8853-885$ p.

15. BRASIL. Lei $N^{\circ} 12.842$, de 10 de julho de 2013. Dispõe sobre o exercício da Medicina. Diário Oficial da União 11 Jul 2013; Seção 1. 1p.

16. Ramos MB, Silva HC; Educação em ciências e em audiovisual: olhares para a formação de leitores de ciências. Cad CEDES. 2014;34(92):51-67. DOI: 10.1590/S010132622014000100004. 